\title{
Relating the probability distribution of a de Broglie wave to its phase velocity
}

\author{
WANG PingXiao ${ }^{1 *}$, WANG JiaXiang ${ }^{2}$, HUO YuKun ${ }^{1}$, SCHEID Werner ${ }^{3} \&$ HORA Heinrich $^{4}$ \\ ${ }^{1}$ Key Laboratory of Applied Ion Beam Physics (Ministry of Education) and Institute of Modern Physics, Department of Nuclear Science and \\ Technology, Fudan University, Shanghai 200433, China; \\ ${ }^{2}$ State Key Laboratory of Precision Spectroscopy and Department of Physics, East China Normal University, Shanghai 200062, China; \\ ${ }^{3}$ Institute for Theoretical Physics, Justus-Liebig-University, Giessen D-35392, Germany; \\ ${ }^{4}$ Department of Theoretical Physics, University of New South Wales, Sydney 2052, Australia
}

Received August 19, 2011; accepted October 24, 2011

\begin{abstract}
We show that the phase velocity in a stationary state of a de Broglie wave can be directly obtained from the probability distribution, i.e. the quantum trajectories, without detailed knowledge of the phase term itself. In other words, the amplitude of a de Broglie wave function describes not only the probability distribution but also the phase velocity distribution. Using this relationship, we comment on two calculations of the Goos-Hänchen shift in de Broglie waves.
\end{abstract}

phase velocity, de Broglie wave, Goos-Hänchen shift

Citation: Wang P X, Wang J X, Huo Y K, et al. Relating the probability distribution of a de Broglie wave to its phase velocity. Chin Sci Bull, 2012, 57: 14941498, doi: 10.1007/s11434-012-5051-0

The importance of phases in physics is self-evident [1], and the phase velocity is a crucial quantity in any description of wave propagation [2,3]. Furthermore, phase velocity and group velocity are particularly important concepts in many applications [4-7]. The statistical interpretation of wave functions is a core concept in quantum mechanics [8,9]. The interpretation can be expressed clearly using only a few words. The interpretation has profound meanings that are not well described by words, and the validity of the interpretation has been confirmed with numerous experiments. The wave function is now an important tool for the description of de Broglie waves, but we can hardly directly measure the wave function itself-let alone its phase and phase velocity. From the form of the wave function, it appears that the probability amplitude and the phase are independent of each other. The statistical probability is related only to the amplitude of the wave function, while the phase velocity can be obtained simply from its phase terms. Based on straightforward algebraic manipulation of the Schrödinger

*Corresponding author (email: wpx@ @udan.edu.cn) and Klein-Gordon equations, however, we deduce a relationship between the distribution of phase velocities and the probability distribution in a stationary state.

The Goos-Hänchen effect in classical optics is a spatial shift on total reflection at an interface. This phenomenon was first observed experimentally by Goos and Hänchen [10]. In the 1960s, Renard and one of us (Hora) studied the Goos-Hänchen shift in matter waves $[11,12]$. Hora expanded the phase term to derive the expression for the GoosHänchen shift, and Renard used probability current conservation. However, their studies led to an unsolved discrepancy between the phase and intensity approaches for calculating the shift. Namely, though their results are almost the same, the latter's expression for the shift has an additional factor which is related to the particle injection angle. This problem was not resolved in the past half century. In addition, Hora's wave bundle is so complex that it cannot be treated with Renard's method using intensity, but Renard's plane wave has nothing to do with Hora's phase term expansion. We deal with this discrepancy using the relationship between the distribution of phase velocities and the 
probability distribution.

\section{Deducing the relation formula}

Waves need not be perfectly periodic with respect to space. As shown in Figure 1, the phase $\varphi$ is the same for $(\boldsymbol{r}, t)$ and $(\boldsymbol{r}+\mathrm{d} \boldsymbol{r}, t+\mathrm{d} t)[2,6]$ provided that

$$
\nabla \varphi \cdot \mathrm{d} \boldsymbol{r}+(\partial \varphi / \partial t) \mathrm{d} t=0
$$

where $\nabla \varphi$ is the gradient of the phase field. Note that the phase velocity does not behave as a vector, so does not obey the resolution rules of vectors. The phase velocity along a direction $\boldsymbol{J}$, and hence its resolution, can be calculated $[2,6,13]$ via $\left(v_{\varphi}\right)_{J}=-(\partial \varphi / \partial t) /(\nabla \varphi)_{J}$, where $(\nabla \varphi)_{J}=$

$\nabla \varphi \cdot \boldsymbol{q}$ is the gradient of the phase field along a unit vector $\boldsymbol{q}$ in the direction $\boldsymbol{J}$. Thus, the minimum phase velocity is given by

$$
v_{p}=-\frac{\partial \varphi / \partial t}{|\nabla \varphi|} .
$$

Note that eq. (2) is none other than the phase velocity mentioned in most references, i.e. the speed with which each of the cophasal surfaces advances [2,3]. If $\partial \varphi / \partial t$ is negative, the cophasal surface advances along the direction of $\nabla \varphi$; otherwise, it advances against the direction of $\nabla \varphi \quad[14,15]$. Unless specified otherwise, the phase velocity in the following refers to the minimum phase velocity.

As in $[16,17]$, we assume a wave function of the form $\psi(\boldsymbol{r}, t)=\psi_{\boldsymbol{r}}(\boldsymbol{r}, t) \mathrm{e}^{i \varphi(\boldsymbol{r}, t)}$, where $\varphi(\boldsymbol{r}, t)$ is the phase of the wave, $\left|\psi_{\boldsymbol{r}}(\boldsymbol{r}, t)\right|$ is the wave amplitude, and $\psi_{\boldsymbol{r}}(\boldsymbol{r}, t)$ and $\varphi(\boldsymbol{r}, t)$ are real functions. Here, the WKB approximation is not needed for our assumption. Substituting $\psi(\boldsymbol{r}, t)$ into the Schrödinger equation,

$$
\left[i \hbar \frac{\partial}{\partial t}+\left(\hbar^{2} / 2 m\right) \nabla^{2}-V(r)\right] \psi(\boldsymbol{r}, t)=0 .
$$

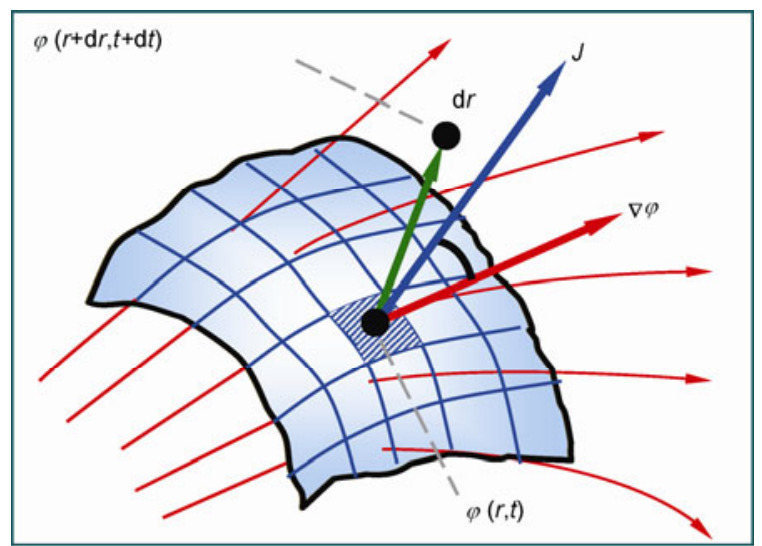

Figure 1 (Color online) Geometry of cophasal surface of a general wave.
Eliminating the $\mathrm{e}^{i \varphi}$ item and collecting the real parts, we obtain [14]

$$
\frac{\partial \varphi}{\partial t}+\frac{\hbar(\nabla \varphi)^{2}}{2 m}+\frac{V(\boldsymbol{r})+U(\boldsymbol{r}, t)}{\hbar}=0,
$$

where $U(\boldsymbol{r}, t)$ is a function of the probability density and can be expressed as

$$
U(\boldsymbol{r}, t)=-\frac{\hbar^{2}}{2 m} \frac{\nabla^{2} \psi_{r}}{\psi_{r}}=-\frac{\hbar^{2}}{4 m}\left(\frac{\nabla^{2} \rho}{\rho}-\frac{1}{2} \frac{(\nabla \rho)^{2}}{\rho^{2}}\right) .
$$

$U(\boldsymbol{r}, t)$ is the so-called "quantum-mechanical" potential in Bohmian Mechanics [17]. From eq. (4), we can obtain the value of the wave vector, which is not a constant but a function of space:

$$
k^{2} \equiv|\nabla \varphi|^{2}=-\frac{2 m}{\hbar}\left[\frac{\partial \varphi}{\partial t}+\frac{V(\boldsymbol{r})+U(\boldsymbol{r}, t)}{\hbar}\right] .
$$

For a stationary state with energy $E, \frac{\partial \varphi}{\partial t}=-\frac{E}{\hbar}=-\omega_{0}$ and $U(\boldsymbol{r}, t)$ is independent of time. Applying eq. (6) to eq. (2), we can get the value of the phase velocity:

$$
v_{p}=\frac{\left|\hbar \omega_{0}\right|}{\sqrt{2 m\left[\hbar \omega_{0}-V(\boldsymbol{r})-U(\boldsymbol{r})\right]}} .
$$

This expression is exactly equivalent to the wave-front speed directly obtained from the generalized de Broglie wavelength and frequency [18]. A prominent feature of this formula is that the phase velocity depends explicitly on the probability distribution and the potential $V(\boldsymbol{r})$. Note that the phase term itself does not appear. We can extend the above results to systems of many bodies by simply rewriting $V(\boldsymbol{r})$ and $U(\boldsymbol{r}, t)$ in the forms for that case [17]. Applying $V(\boldsymbol{r})=0$ and $U(\boldsymbol{r})=0$ to the relationship yields the phase velocity of the de Broglie wave for a free particle, $v_{p}=\hbar \omega_{0} / \sqrt{2 m E}=\omega_{0} / k_{0}$. This result is the same as for the original definition [19]. From the probability current density $\boldsymbol{j}=\rho \boldsymbol{v}=\frac{\rho \hbar}{m} \nabla \varphi$, we get the mean velocity of the particle, $|\boldsymbol{v}|=2 v_{p}$.

To verify the above relationship, we consider a typical hydrogen-like electron state with nonzero angular momentum [17]:

$$
\psi(\boldsymbol{r}, t)=R_{n \ell}(r) P_{\ell}^{\mu x}(\cos \theta) \mathrm{e}^{i\left(\alpha-E_{n} t+\hbar \mu \phi\right) / \hbar},
$$

where $\alpha$ is a constant, $r$ is the radius from the atom's center, and $\theta$ and $\phi$ are the colatitude and azimuthal polar angles, respectively. Because $R_{n \ell}(r) P_{\ell}^{m}(\cos \theta)$ is a real function, it is easy for us to calculate the wave vector from the phase term $\left(\alpha-E_{n} t+\hbar \not h \phi\right) / \hbar$ using

$$
k \equiv \nabla \varphi=\frac{\hbar \not h}{r \sin \theta} \boldsymbol{e}_{\phi},
$$


where $\boldsymbol{e}_{\phi}$ is a unit vector in the direction of $\phi$. The phase velocity is

$$
v_{p} \equiv \frac{|\partial \varphi / \partial t|}{|\nabla \varphi|}=\left|\frac{E_{n} r \sin \theta}{\hbar m}\right|,
$$

where $E_{n}=-\frac{Z^{2} m e^{4}}{2 \hbar n^{2}}$. If we apply $\psi_{r}=R_{n \ell}(r) P_{\ell}^{m}(\cos \theta)$ and $V(\boldsymbol{r})=-\frac{Z e^{2}}{r}$ to eqs. (5) and (7), we obtain exactly the same result. The electron mean velocity is

$$
\boldsymbol{v}=\frac{\hbar}{m} \nabla \varphi=\frac{\hbar}{m} \frac{\not h}{r \sin \theta} \boldsymbol{e}_{\phi} .
$$

Here, zero angular momentum ( $\not h=0$, or $k \equiv|\nabla \varphi|=0)$ means that every point in the phase field has same phase at the same time. In other words, the phase velocity is infinite, but the mean electron velocity is $v=\frac{\hbar}{m} \nabla \varphi=0$.

\section{Evaluating the Goos-Hänchen shift}

We know that the wave's propagation is closely related to its phase velocity. Renard and Hora used Snell's law together with the particles' mean velocity to calculate the Goos-Hänchen shift $[11,12]$. If the de Broglie waves, either reflected or refracted at a potential barrier, are treated similarly to optical waves that are either reflected or refracted at a media interface, then it is important to look at the phase velocity. In principle, the methods should be equivalent to each other if their treatments are correct. Then the inconsistencies may be due to considering different physical objects.

Hora used a wave bundle consisting of a family of superposed plane waves with angles of incidence from $\alpha_{0}-\varepsilon$ to $\alpha_{0}+\varepsilon$, where the potential of incidence region is a constant, as shown in Figure 2. $\varepsilon$ is a small parameter,

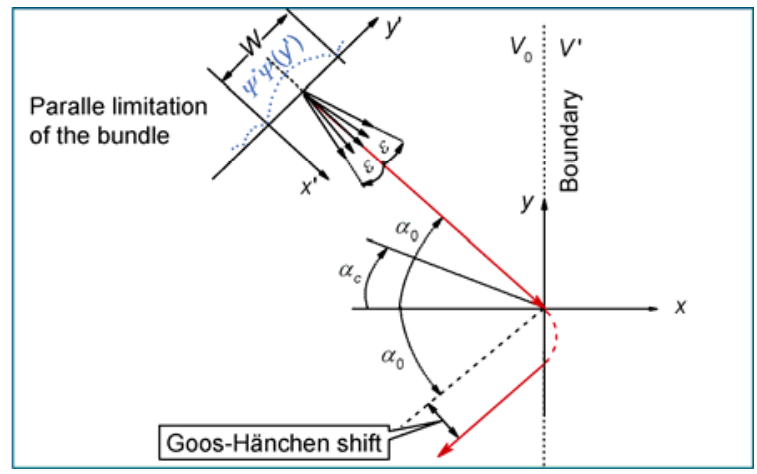

Figure 2 (Color online) Coordinate axes used for treating the Goos-Hänchen shift of a de Broglie wave bundle in Hora's method. The constant potential $V_{0}$ is in the region where $x<0$, and $V^{\prime}$ is in the region where $x>0$. Assume $E>V^{\prime}>V_{0}$. and the angle of incidence $\alpha_{i}$ is always larger than the critical angle of total reflection $\alpha_{c}$. Obviously, it is difficult to obtain this wave bundle's separate phase term. The waves have been chosen so that the cross section of the bundle written as the probability density with respect to a coordinate $y^{\prime}[20]$ is

$$
\rho=\psi^{*} \psi \sim \frac{\left[\sin \left(2 \pi y^{\prime} / W\right)\right]^{2}}{y^{\prime 2}} .
$$

The bounds of the bundle given by the first lateral minima of the $\psi^{*} \psi$ function are exactly parallel and define the width $W=\lambda / \varepsilon$ of the bundle, where $\lambda$ is the de Broglie wavelength. As a result, $\rho$ is independent for coordinates $x^{\prime}$ and $z^{\prime}$. According to eq. (7), we get the wave bundle's phase velocity distribution in terms of the $x^{\prime} y^{\prime}$ coordinates:

$$
v_{p}=\frac{\frac{m|\boldsymbol{v}|^{2}}{2}+V_{0}}{\sqrt{m^{2}|\boldsymbol{v}|^{2}+2 \hbar^{2}\left\{\frac{1-\left[\operatorname{ctg}\left(2 \pi y^{\prime} / W\right)\right]\left(2 \pi y^{\prime} / W\right)}{y^{\prime 2}}-2(\pi / W)^{2}\right\}}}
$$

The phase velocity of this wave bundle depends on the space coordinates; therefore, the cophasal surfaces must be distorted during propagation. This behavior is completely different from the case of a plane wave of light. In the center region $\left(y^{\prime} \rightarrow 0\right.$ ) of the wave bundle, we get

$$
v_{p} \rightarrow \frac{\frac{m|\boldsymbol{v}|^{2}}{2}+V_{0}}{\sqrt{m^{2}|\boldsymbol{v}|^{2}+\frac{4}{3} \hbar^{2}(\pi / W)^{2}}} .
$$

Instead, Renard used a plane wave for the derivation, and the potential of incidence region is also a constant, $V_{0}$ [12]. According to eq. (7), we get a relationship between the mean particle velocity $\boldsymbol{v}$ and the phase velocity of its plane matter wave:

$$
v_{p}=\frac{|\boldsymbol{v}|}{2}+\frac{V_{0}}{m|\boldsymbol{v}|} .
$$

As is the case for a plane wave of light, the phase velocity of the plane wave in a stationary potential is independent of the space coordinates, meaning that the cophasal surfaces will not be distorted during propagation.

From eq. (14) we find that, even at the center of Hora's wave bundle, the relationship between the phase velocity and mean particle velocity is different from that of a plane wave in Renard's method. Not surprisingly, the results differ because the behavior of the cophasal surfaces in each of these methods is very different. This means that the Goos-Hänchen shift of a matter wave also depends on its wave mode. This may be helpful in designing and modulating quantum microstructure electronic devices [21].

Using Hora's wave bundle in the stationary-phase approach, which is somewhat different from Hora's method, 
yields the same result as in his method [21]. A good experimental comparison with Renard's result has recently been done [22], but we anticipate more sophisticated experiments to distinguish their difference. Here we simply consider non-spinning particles. Actually, the Goos-Hänchen shift of a de Broglie wave also depends on the spin [23,24].

\section{Case of a Lorentz covariant wave equation}

A similar relationship can be obtained even if using a Lorentz covariant wave equation. We first simply consider the Klein-Gordon equation for a single charged particle in an electromagnetic field $A_{\mu}(\boldsymbol{r})=\left(A_{x}, A_{y}, A_{z}, i \mathcal{V} / c\right)$ [25-27]:

$$
\left[\left(\partial_{\mu}-\frac{i e}{\hbar} A_{\mu}\right)^{2}-\frac{m^{2} c^{2}}{\hbar^{2}}\right] \psi(\boldsymbol{r}, t)=0
$$

where $\partial_{\mu} \equiv \frac{\partial}{\partial x_{\mu}}$, and $x_{\mu}=(\boldsymbol{r}, i c t)$. Substituting $\psi(\boldsymbol{r}, t)$

into the Klein-Gordon equation, eliminating the $\mathrm{e}^{i \varphi}$ item and collecting the real parts, we obtain

$$
\begin{aligned}
(\nabla \varphi \cdot \nabla \varphi)= & \partial_{\mu}^{2} \psi_{r}+\frac{1}{c^{2}}\left(\frac{\partial \varphi}{\partial t}\right)^{2}-\frac{m^{2} c^{2}}{\hbar^{2}} \\
& -\frac{e^{2}}{\hbar^{2}}\left(A_{x}^{2}+A_{y}^{2}+A_{z}^{2}-\frac{\mathcal{V}^{2}}{c^{2}}\right) \\
& +\frac{2 e}{\hbar}\left(A_{x} \frac{\partial \varphi}{\partial x}+A_{y} \frac{\partial \varphi}{\partial y}+A_{z} \frac{\partial \varphi}{\partial z}+\frac{\mathcal{V}}{c^{2}} \frac{\partial \varphi}{\partial t}\right) .
\end{aligned}
$$

Now consider the specific case of an electromagnetic field $A_{\mu}(\boldsymbol{r})=(\boldsymbol{A} \equiv 0, i \mathcal{V} / c)$. According to eqs. (2) and (17), the phase velocity is

$$
v_{p}=\frac{\left|\hbar \omega_{0}\right|}{\sqrt{\frac{1}{c^{2}}\left[\left(\hbar \omega_{0}-e \mathcal{V}\right)^{2}-m^{2} c^{4}\right]-2 m \tilde{U}(\boldsymbol{r}, t)}},
$$

where $\tilde{U}(\boldsymbol{r}, t)=-\frac{\hbar^{2}}{2 m} \frac{\partial_{\mu}^{2} \psi_{r}}{\psi_{\boldsymbol{r}}}$ and $(E-e \mathcal{V})^{2}=\left(\hbar \omega_{0}-e \mathcal{V}\right)^{2}$ $=c^{2} \hbar^{2} k^{2}+m^{2} c^{4}$. For a stationary state, $\frac{\partial \psi_{r}}{\partial t}=0$, i.e. $\tilde{U}(\boldsymbol{r}, t)=U(\boldsymbol{r})=-\frac{\hbar^{2}}{2 m} \frac{\nabla^{2} \psi_{\boldsymbol{r}}}{\psi_{\boldsymbol{r}}}$. An electromagnetic field of the form $A_{\mu}^{\prime}=\left(A^{\prime} \neq 0, i \mathcal{V}^{\prime} / c\right)$ can be changed to the form $A_{\mu}=(\boldsymbol{A} \equiv 0, i \mathcal{V} / c)$ via a Lorentz transformation, with Lorentz factor $\gamma_{\mu}=\mathcal{V}^{\prime} / \sqrt{\mathcal{V}^{\prime 2}-c^{2} A_{\mu}^{\prime 2}}, \mu=x, y, z \quad$ [28]. Thus, we can get the phase velocity of a de Broglie wave in $A_{\mu}^{\prime}$ by an inverse Lorentz transformation. If we apply $\mathcal{V}=0$ and $\tilde{U}(\boldsymbol{r})=0$ to the relationship eq. (18), we again obtain the phase velocity of a free particle de Broglie wave: $v_{p}=\omega_{0} / k_{0}$.
Putting aside the problems that the Klein-Gordon equation meets in quantum mechanics, it is noteworthy that the Klein-Gordon equation of a massless particle $(m=0)$ in free space is precisely the classical wave equation [28]. We can therefore deal similarly with electromagnetic waves and sound waves in a uniform medium.

\section{Summary and discussion}

The probability amplitude and instantaneous phase of a point on a wave function are considered hard to measure. Using the relationship we have mentioned, we can obtain the phase velocity distribution by calculating or measuring the potential distributions and the probability density. This approach is especially useful for wave functions with complicated forms, where it is difficult to obtain an analytical form for the phase. Measuring the optical phase distribution in a small beam is very difficult [29], especially when the difference between the phase velocity and the standard light speed $c$ is tiny. Using a nonlinear optical approach to measure the phase velocity indirectly is also difficult and needs an approximation [30,31]. In [32], we discussed the case of an electromagnetic wave in vacuum and considered the stationary fundamental Hermite-Gaussian mode of a laser beam in vacuum. We compared two approaches for obtaining the velocity, the above relation (eq. (18)) and directly evaluating the phase terms, and found the approaches equivalent to the lowest order of a paraxial approximation.

We would like to point out that the concept of "quantum-mechanical" potential is not essential to our result; nevertheless, the new relationship may help us understand more about the quantum trajectory method applied in reactive scattering [33], photodissociation and femtochemistry [34]. The de Broglie wave of nucleons at internal total reflection on the nuclear surface potential may also provide insight into Wigner scattering [35].

A special word of thanks goes to Prof. G. L. Long (Tsinghua University, China) and Prof. Roger Hutton (Lund University, Sweden) for their useful comments. One of the authors (P.X.W.) was supported by the Program for New Century Excellent Talents in University, Chinese Ministry of Education. This work was partly supported by the National Natural Science Foundation of China (10975036).

1 Yang C N. Complex phases in quantum mechanics. In: Proceedings of the 2nd International Symposium on Foundation of Quantum Mechanics, Tokyo, 1986. 181-184

2 Born M, Wolf E. Principles of Optics. 7th ed. Cambridge: Cambridge University Press, 1999. 16-19

3 Brillouin L. Wave Propagation and Group Velocity. New York: Academic, 1960. 1-16

4 Esarey E, Sprangle P, Pilloff M, et al. Theory and group velocity of ultrashort, tightly focused laser pulses. J Opt Soc Am B, 1995, 12: 1695-1703

5 Gao F, Xu, J J, Zhang, G Q, et al. Nonlinear optical properties and superluminal propagation in the ruby. Chin Sci Bull, 2010, 55: 473-477 
6 Wang P X, Ho Y K, Yuan X Q, et al. Vacuum electron acceleration by an intense laser. Appl Phys Lett, 2001, 78: 2253-2255

7 Nimtz G, Haibel A. Basics of superluminal signals. Ann Phys, 2002, 11: 163-171

8 Born M. For the quantum mechanics of the shock processes. Z Phys, 1926, 37: 863-867

9 Ballentine L E. The statistical interpretation of quantum mechanics. Rev Mod Phys, 1970, 42: 358-381

10 Goos F, Hänchen H. Ein neuer und fundamental versuch zur totalreflexion. Ann Phys, 1947, 436: 333-346

11 Renard R H. Total reflection: A new evaluation of the Goos-Hänchen shift. J Opt Soc Am, 1964, 54: 1190-1197

12 Hora $H$. Zur seitenversetzung bei der totalreflexion von matteriewellen. Optik, 1960, 17: 409-415

13 Strumia A. Waves, particles, and field dynamics. J Math Phys, 2006, 47: 083509

14 Huang Y S. The invariance of the phase of waves among inertial frames is questionable. Europhys Lett, 2007, 79: 10006

15 Gjurchinovski A. Is the phase of plane waves a frame-independent quantity? Europhys Lett, 2008, 83: 10001

16 Madelung E. Quantum theory in hydrodynamic form. Z Phys, 1927, 40: 322-326

17 Bohm D. A suggested interpretation of the quantum theory in terms of 'Hidden' variables. I. Phys Rev, 1952, 85: 166-179

18 Holland P R. The Quantum Theory of Motion. Cambridge: Cambridge University Press, 1993. 87-88

19 De Broglie L. Wave and quanta. Nature, 1923, 112: 540

20 Carter J L, Hora H. Total reflection of matter waves: The Goos-Hänchen effect for grazing incidence. J Opt Soc Am, 1971, 61: $1640-1645$

21 Wilson D W, Glytsis E N, Gaylord T K. Electron wave-guiding characteristics and ballistic current capacity of semiconductor quantum slabs. IEEE J Quant Electron, 1993, 29: 1364-1382
22 Haan V O, Plomp J, Rekveldt T M, et al. Observation of the Goos-Hänchen shift with neutrons. Phys Rev Lett, 2010, 104: 010401

23 Beenakker C W J, Sepkhanov R A, Akhmerov A R, et al. Quantum Goos-Hänchen effect in graphene. Phys Rev Lett, 2009, 102: 146804

24 Huang J H, Duan Z L, Ling H Y, et al. Goos-Hänchen-like shifts in atom optics. Phys Rev A, 2008, 77: 063608

25 Klein O. Quantum theory and five dimensional theory of relativity. Z Phys, 1926, 37: 895-906

26 Klein O. Electrodynamics and wave mechanics from the standpoint of the correspondence principle. Z Phys, 1927, 41: 407-442

27 Gordon W. The Compton effect according to the Schrödinger's theory. Z Phys, 1926, 40: 117-133

28 Jackson J D. Classical Electrodynamics. 3rd ed. New York: John Wiley \& Sons Inc., 1999. 526-527

29 Phillips P L, Knight J C, Pottage J M, et al. Direct measurement of optical phase in the near field. Appl Phys Lett, 2000, 76: 541-543

30 Wang K, Qian L J, Qiu P, et al. Phase-velocity measurement of a tightly focused Gaussian beam by use of sum frequency generation. Appl Phys Lett, 2008, 92: 121114

31 Wang K, Qian L J, Zhu H Y. Soliton pulse compression through cascaded quadratic nonlinearity in difference-frequency generation. Chin Sci Bull, 2008, 53: 1941-1945

32 Chen Z, Ho Y K, Wang P X, et al. A formula on phase velocity of waves and application. Appl Phys Lett, 2006, 88: 121125

33 Terlecki G, Grün N, Scheid W. Solution of the time-dependent Schrödinger equation with a trajectory method and application to $\mathrm{H}^{+}-\mathrm{H}$ scattering. Phys Lett A, 1982, 88: 33-36

34 Goldfarb Y, Degani I, Tannor D J. Bohmian mechanics with complex action: A new trajectory-based formulation of quantum mechanics. $\mathrm{J}$ Chem Phys, 2006, 125: 231103

35 Hora H. From laser produced Debye layers in plasmas to a theory of nuclear forces and quark-gluon plasmas. Laser Part Beams, 2006, 24: $35-40$

Open Access This article is distributed under the terms of the Creative Commons Attribution License which permits any use, distribution, and reproduction in any medium, provided the original author(s) and source are credited. 